Artigo de revisão

\title{
ESTRESSE OXIDATIVO E O SEU IMPACTO NO ENVELHECIMENTO: UMA REVISÃO BIBLIOGRAFICA
}

\author{
Autores: Letícia Porsch', Luisa Amábile Wolpe Simas ${ }^{2,1}$, Rodrigo Otávio Chybior Granzoti ${ }^{3}$ \\ ${ }^{1}$ Pós-graduanda pelo Instituto Ana Paula Pujol, Camboriú, Santa Catarina, Brasil. \\ ${ }^{2}$ Nutricionista. Pós-graduada em Nutrição Clínica pela Universidade Federal do Paraná. Mestre em Medicina Interna pela \\ Universidade Federal do Paraná, Paraná, Brasil. \\ ${ }^{3}$ Biólogo, acadêmico do curso de Nutrição Faculdade Paranaense, Paraná, Brasil.
}

\section{Informações do artigo}

Palavras Chave:

Estresse oxidativo; envelhecimento; senescência; espécies reativas de oxigênio.

\section{Resumo}

A elevação na expectativa de vida reflete num envelhecimento da população, e por consequência em um incremento nas doenças associadas a ela. Nos últimos anos houve um aumento nos estudos vinculados ao tema o que ocasionou a elaboração de muitas teorias destinadas a explicar este processo dentre elas está a Teoria dos Radicais Livres, atualmente a mais aceita. Esta teoria defende a hipótese de que durante o metabolismo aeróbico normal, o oxigênio sofre redução formando espécies reativas do oxigênio, os quais se somariam aos demais Radicais Livres advindos de diferentes mecanismos geradores. O organismo para defender-se da ação lesiva desses Radicais Livres, conta com diferentes sistemas de defesa antioxidante. Porém, ao ocorrer desequilíbrio na formação de Radicais Livres e nesta defesa, há um incremento no número dessas espécies reativas, etapa conhecida como estresse oxidativo. Nessas circunstâncias ocorreria uma perda gradual da capacidade funcional da célula, repercutindo no envelhecimento. Contudo, conclusões definitivas acerca da origem e desenvolvimento do envelhecimento, requerem maiores estudos, uma vez que esse fenômeno pode não ter uma causa fundamental.

\section{Article ID}

Keywords:

Oxidative stress; aging;

senescence; Oxigen-reac-

tive species.

\section{Abstract}

Various cosmetic formulations use actives to attenuate the signs of photoaging, substances such as alpha hydroxy acids, beta hydroxy acids and polyhydroxy acids. This literature review discusses the molecular aspects

\section{${ }^{1}$ Autor correspondente:}

Luisa Amábile Wolpe Simas: e-mail: luisa.fies@hotmail.com - https://orcid.org/0000-0003-2323-5665

DOI: https://doi.org/10.31415/bjns.v2i2.53 - Artigo recebido em: 14 de fevereiro de 2019 ; aceito em 18 de abril de 2019 ; publicado em 29 de maio de 2019. Brazilian Journal of Natural Sciences, Vol. 2, N.2, maio 2019. Disponível online a partir de 29 de maio de 2019, ISSN 2595-0584. www.bjns.com.br - Todos os autores contribuíram igualmente com o artigo. Este é um artigo de acesso aberto sob a licença CC - BY: http://creativecommons.org/licenses/by/4.0 
of polyhydroxy acid lactobionic acid and its effects on photoaged skin, according to some clinical data were observed fine lines and wrinkles around the eyes, flaccidity, dilated ostia, roughness and luminosity on the face. In its histology the effects of UV radiation on skin changes. As a result, lactobionic acid has been shown to be effective in improving clinical signs of photoaged skin.

\section{Introdução}

O envelhecimento é um fenômeno universal inevitável, biológico que afeta todos os organismos multicelulares. Embora diferentes hipóteses têm sido propostas para explicar os mecanismos celulares e moleculares do envelhecimento, estudos recentes tornam-se cada vez mais claros que o envelhecimento está relacionado ao acúmulo de danos moleculares, dando origem a uma teoria unificada do envelhecimento. Entre as reações que contribuem para este dano, as reações de radicais livres e outras espécies reativas de oxigênio são consideradas a principal razão, além das reações de metabolitos, tais como os açúcares e os aldeídos reativos e erros espontâneos em processos bioquímicos [1].

Há vários séculos que o homem se interroga sobre o processo do envelhecimento e procura conhecer quais as suas causas, tendo surgido ao longo dos anos várias teorias para o explicar, entre elas está a teoria do envelhecimento biológico, a teoria da ação do estresse oxidativo que tem sido uma das mais aceitas e estudadas[2].

Atualmente, existe um grande interesse por profissionais da área de saúde sobre as causas do envelhecimento humano e sobre os fatores endógenos e exógenos que podem acelerar ou retardar no processo de senescência. Tal questionamento torna-se

de indubitável discussão quando nos referimos às espécies reativas de oxigênio (EROS), pois estas são as principais substâncias relacionadas à oxidação celular e tissular, e torna-se alvo de pesquisas científicas que as demostrem como principal causa do envelhecimento humano. Estas também são alvo das indústrias de fármacos, cosméticos e alimentos, a fim de retardar o processo de oxidação celular [3]

Cerca de 5\% do oxigênio utilizado pelos organismos, via metabolismo oxidativo, não é utilizado nos ciclos mitocondriais que produzem energia. Esse oxigênio excedente tende a perder dois elétrons na sua última camada, produzindo o radical superóxi- do ou, também, por ações enzimáticas e metabólicas adicionais, pode formar outros tipos de moléculas desemparelhadas de oxigênio, que são genericamente conhecidas como EROS. Por serem moléculas altamente reativas, o organismo controla a sua degradação através de dois sistemas antioxidantes integrados: um endógeno enzimático, diretamente relacionado à degradação do superóxido em água, e outro exógeno não enzimático, no qual compostos antioxidantes presentes na dieta atuam sobre as EROS produzidas pelo organismo [4].

Deste modo, o estresse oxidativo é visto como um desbalanço entre a produção de EROS e sua degradação pelos antioxidantes segundo a necessidade de cada célula. Nestes termos, o acúmulo ou o descontrole da produção de EROS, ainda que não seja, necessariamente, considerado um fator causal, mas atuando mais com um fator modulador dos mecanismos envolvidos no processo de envelhecimento, está associado a um grande número de condições patológicas [4].

O presente estudo tem como objetivo a elaboração de uma revisão bibliográfica para avaliar o impacto do estresse oxidativo no envelhecimento humano.

\section{Metodologia}

Trata-se de uma revisão sistemática de literatura, realizada em bases de dados eletrônicas (Pubmed, Google Acadêmico, SciELO), em periódicos nacionais e internacionais, busca por artigos de revisão, artigos em humanos e roedores, artigos originais, pelas palavras-chave: estresse oxidativo, envelhecimento, senescência, espécies reativas de oxigênio e os seus respectivos termos em inglês. A seleção dos artigos teve como critérios de inclusão: amostras contendo humanos em seus estudos; estudos nacionais e internacionais, artigos originais e de revisão com publicação entre os anos de 2011 a 2018.

\section{Resultado e discussão}


O envelhecimento é um processo biológico heterogêneo, progressivo, deletério e irreversível no qual ocorrem modificações que reduzem a capacidade de adaptação do indivíduo ao meio ambiente, permitindo o surgimento das doenças crônicas, como a obesidade, que gera, por sua vez, complicações secundárias como hipertensão, diabetes, doenças cardiovasculares e osteoartrites, com impacto sobre a saúde e a qualidade de vida do idoso, aumentando a vulnerabilidade e risco de morte [3].

O envelhecimento é um processo altamente complexo porque a degeneração celular envolve milhares de mecanismos e processos diferentes. Durante o envelhecimento, as reações redox metabólicas celulares induzem alterações genéticas e bioquímicas prejudiciais [5].

Marcas do envelhecimento têm sido propostas, como a senescência celular eo desgaste dos telômeros, que se acredita representem mecanismos-chave pelos quais o envelhecimento contribui para o desenvolvimento de condições relacionadas à idade. Uma das características é a disfunção mitocondrial, que pode promover distúrbios relacionados à idade, em parte, através do aumento dos níveis de espécies reativas de oxigênio [6].

O envelhecimento é acompanhado por um aumento do estresse oxidativo [7], aonde os níveis aumentados de ROS podem resultar em dano oxidativo, que é um mecanismo pelo qual as ROS podem promover doenças relacionadas com a idade. No processo de produção de energia mitocondrial o oxigênio é parcialmente convertido em espécies reativas de oxigênio (EROS) [6]. Quando a produção de EROS excede sua degradação, denominamos estresse oxidativo. Nosso organismo possui defesas antioxidantes naturais que neutralizam as EROS [7].A glutationa é um importante antioxidante intracelular aonde pode notar um aumento relacionado à idade na quantidade de glutationa oxidada em relação à reduzida, consistente com um aumento relacionado à idade nas EROS [6].

O estresse oxidativo é um desequilíbrio entre a produção de EROS presente nas células e a capacidade biológica para desintoxicar os intermediários reativos ou reparar os danos causados. Ele desempenha um papel chave no desenvolvimento de várias doenças cardiovasculares. Existem muitos fatores associados ao estresse oxidativo, que conduzem ao desenvolvimento destas doenças. Um dos principais fatores é a superprodução de EROS, juntamente com a diminui- ção da biodisponibilidade de óxido nítrico que reduz a capacidade antioxidante na vasculatura $[\mathbf{8}]$.

Muitas vezes a produção de EROS excede a capacidade de remoção causando assim danos no DNA, proteínas e fosfolipídios dos diversos tipos de células, contribuindo para o aparecimento de doenças degenerativas relacionadas com a idade. $\mathrm{O}$ estresse oxidativo é gerado continuamente no nosso organismo fruto de eventos estocásticos e pode ser atenuado ou agravado mediante os comportamentos da vida diária. Entre estes, a literatura tem salientado a influência marcante do estilo de vida ativo, da dieta, dos hábitos tabácicos, do consumo de álcool, da exposição a radiações e a substâncias toxicas [7].

A teoria dos radicais livres do envelhecimento postula que a produção de espécies reativas de oxigênio intracelulares é o principal determinante da vida. Atualmente, o estresse oxidativo e mecanismos de defesa antioxidante prejudicada se acredita serem os principais contribuintes para o processo de envelhecimento cardiovascular. O estresse oxidativo desenvolve-se como uma consequência da geração excessiva de EROS, por enzimas tais como a NADPH-oxidase, sintase do óxido nítrico desacoplado, e oxidase de xantina, por a cadeia de transporte eletrônico mitocondrial, e, como resultado está redução da capacidade antioxidante [9].

Quando um radical livre não encontra nenhum outro radical livre para se ligar na tentativa de obter estabilidade, eles captam elétrons de outras moléculas saudáveis. A molécula que perdeu o elétron se transforma então em outro radical livre, iniciando-se uma reação em cadeia, que danificará muitas células, podendo ter caráter ilimitado, se não houver a intervenção dos antioxidantes. Este processo é chamado de oxidação, e provoca morte celular [10]. Por isso os efeitos benéficos da alimentação na prevenção do estresse oxidativo têm sido atribuídas à presença de antioxidantes [11].

Os antioxidantes podem ser produzidos pelo corpo ou absorvidos pela dieta. Dentre os que são produzidos pelo corpo os mais comumente encontrados são as enzimas Glutationa peroxidasse (GPx), a Catalase (CAT) e a Superóxido dismutase (SOD), e os peptídeos Glutationa (GSH), Entre os absorvidos pela dieta destacam-se o $\alpha$-tocoferol (vitamina-E), $\beta$-caroteno (provitamina-A), ácido ascórbico (vitamina-C), selênio e compostos fenólicos como os flavonoides e poliflavonoides. Todos estes exercem ações sinérgi- 
cas na limpeza dos radicais e por sua vez desempenham um papel importante na prevenção de muitas doenças como, por exemplo, o câncer, a aterosclerose, acidente vascular cerebral, a artrite reumatoide, a neurodegeneração e diabetes [7]. Ao passar dos anos, a concentração de radicaislivres aumenta, e nossas defesas naturais antioxidantes falham, com isso o uso de compostos antioxidantes encontrados na dieta é um importante mecanismo de defesa contra os radicais livres. Para evitar que ocorra o estresse oxidativo, é imprescindível que haja um equilíbrio entre radicais livres e antioxidantes no nosso organismo. Por isso é de extrema importância o consumo de alimentos que contenham vitaminas antioxidantes na nossa alimentação, para evitar que ocorram os danos provocados pelo desequilíbrio entre esses dois sistemas e para que isso não acelere o processo de envelhecimento cutâneo [10].

Estudos epidemiológicos recentes sugerem que os carotenoides ou alimentos ricos em carotenoides são protetores contra um declínio da força muscular em indivíduos idosos. Desta forma, os carotenoides têm a função de proteger contra o estresse oxidativo por têmpera oxigênio atómico, atuando na eliminação dos radicais livres, e inibindo a peroxidação lipídica [11].

Quando a geração de EROS excede a sua neutralização por enzimas antioxidantes, uma condição de estresse oxidativo surge e níveis elevados de EROS podem causar danos nas macromoléculas como DNA mitocondrial e nuclear, proteínas, polissacarídeos, ácidos e lipídeos, levando a perturbações que estão relacionadas na patogênese de uma série de doenças neurodegenerativas e morte celular. O acumulo de lesões oxidativas com a idade ocorre por três fatores, se a célula produzir mais EROS do que remove, se houver uma diminuição das defesas antioxidantes, ou uma redução na eficiência de reparação das moléculas danificadas [7].

A exposição de células ao estresse não-letal recorrente ou crônico poderia contribuir para um aumento no acúmulo de células senescentes induzidas pelo estresse, acelerando o envelhecimento dos tecidos. Acredita-se que as células senescentes se acumulam com a idade parcialmente devido à sua resistência à apoptose, não se pode excluir que pelo menos algumas delas são eliminadas pelo sistema imunológico, ou que em certas circunstâncias elas podem morrer. Embora o papel causal das espécies reativas de oxigê- nio (ROS) no envelhecimento seja discutível, o paradigma assumindo que o estresse oxidativo e as ROS produzidas pelas mitocôndrias desempenham um papel importante na senescência celular tem sido apoiado. O peróxido de hidrogênio foi o primeiro fator usado para mostrar senescência induzida por estresse oxidativo [12].

A disfunção mitocondrial é um importante gatilho para a senescência celular, o que contribui para o envelhecimento, além de outros estímulos de pró senescência. Embora esteja claro que as mitocôndrias disfuncionais se acumulam em células senescentes e que esta é uma força motriz importante para o envelhecimento acelerado, é menos compreendido como as mitocôndrias se tornam disfuncionais durante $o$ processo de envelhecimento. Os defeitos mitocondriais que se acumulam nas células mais antigas variam de um aumento da massa mitocondrial, diminuição do acoplamento respiratório, produção de ATP menos eficiente durante a respiração, perda da função do complexo respiratório I e aumento da produção de ROS mitocondrial [13].

Várias décadas após a teoria original do estresse oxidativo e envelhecimento, nossa compreensão do estresse oxidativo avançou significativamente. Embora um grande corpo de evidências revele univocamente os papéis potenciais do estresse oxidativo nas degenerações celulares ligadas a uma ampla gama de doenças degenerativas relacionadas ao envelhecimento, estudos recentes mostram os importantes papéis do estresse oxidativo na defesa imunológica, sinalização molecular e sobrevivência celular [5].

A complexidade do processo de envelhecimento e o esforço para compreender os seus alicerces moleculares, orientam o foco para abordagens para melhorar o tempo de vida e vitalidade do organismo. Apesar da transição da curiosidade da cultura celular para um potencial regulador do câncer e do envelhecimento, a senescência celular permanece enigmática e continua a levantar uma variedade de questões complexas. $\mathrm{O}$ estresse oxidativo desempenha um papel importante em várias vias que levam à senescência celular e ao fenótipo secretor associado. Embora inicialmente pretenda ser uma resposta protetora à agressão oncogênica, a senescência celular induzida por agentes danificadores de DNA e ROS, pode potencialmente provocar efeitos prejudiciais e pode levar a muitas doenças relacionadas à idade [14].

Um dos fatores que parece se correlacionar com a 
diminuição do estresse oxidativo é a adoção de uma restrição calórica, sem desnutrição. Várias pesquisas têm sido realizadas acerca disto a fim de averiguar quais seriam efetivamente os benefícios de uma dieta hipocalórica e a influência de uma alimentação saudável na promoção da longevidade. Em pesquisas realizadas a partir de células neuronais in vitro, a restrição calórica desempenhou um fator relevante na redução de marcadores de estresse oxidativo, que eram menores em culturas de células neuronais tratadas com soro com restrição calórica em comparação com as células tratadas com soro rico ad libitum. Estudos apontam que a restrição calórica tende a aumentar a expressão de antioxidantes, tendendo a exibir maior quantidade de vitamina E, e Coenzima Q10 nas membranas celulares cerebrais [3].

\section{Considerações finais}

O envelhecimento é um fenômeno complexo que envolve inúmeros fatores, o que consequentemente provoca a elaboração de várias hipóteses e teorias voltadas para explicar esse processo.

O estresse oxidativo causa danos às células, sendo considerado um dos fatores preponderantes do envelhecimento. Muitos estudos apontam os benefícios oferecidos pela dieta de restrição calórica na redução do estresse oxidativo, embora não se pode afirmar que os efeitos de uma dieta de poucos nutrientes possam efetivamente promover a longevidade, sendo necessários novos estudos que comprovem os benefícios. Além disso, por meio de estudos, doenças associadas ao envelhecimento têm sido relacionadas à ação das espécies reativas de oxigênio, como as demências, mas sem conclusões sobre causalidade entre os dois.

Por tanto, envelhecer é um processo natural pelo qual todas as pessoas passam na vida e não tem como evitar, além das funções fisiológicas, a pele exerce papel na autoestima do ser humano. Todos querem viver muito e manter uma aparência jovial, por isso devemos ter bons hábitos alimentares e um estilo de vida saudável, esses fatores amenizam as marcas do tempo, e são hábitos fundamentais para a prevenção do envelhecimento cutâneo, além de aumentar as chances de viver mais e sentir-se melhor e mais jovem. Uma alimentação rica em antioxidantes como as vitaminas $\mathrm{A}$, vitamina $\mathrm{C}$ e a vitamina $\mathrm{E}$, é a melhor forma de prevenção aos efeitos nocivos dos radicais livres em excesso, pois as vitaminas atuam inibindo a ação destes radicais. Evitar exposições excessivas ao sol também faz parte da prevenção do envelhecimento cutâneo e formação de radicais livres, mas apenas a reposição das vitaminas não terá o efeito desejado se não forem associadas a uma alimentação balanceada, horas adequadas de sono e exercícios físicos moderados. Pelo contrário, alguns antioxidantes em excesso podem inclusive produzir mais radicais livres.

\section{Referências}

1. SADOWSKA-BARTOSZ I., BARTOSZ G. Effect of Antioxidants Supplementation on Aging and Longevity. Hindawi Publishing Corporation BioMed Research International, Article ID 404680, pg 1-17, 2014.

2. TEIXEIRA J., FEIO M., FIGUEIRA M.L. O Papel do Stress Oxidativo no Envelhecimento e na Demência. Revista do Serviço de Psiquiatria do Hospital Professor Doutor Fernando Fonseca, EPE, vol. 12, nº 1, pg. 1-15, 2014.

3. LEITE L.M.G.S., BRASIL R.L.F; MARQUES T.M.A.; CRUZ J.B.; CATÃO C.D.S.; Estresse Oxidativo e Envelhecimento Humano: Uma Revisão Sistemática. Anais CIEH, vol. 2, n.1. ISSN 2318-0854, 2015.

4. GOTTLIEB M. G. V., MORASSUTTI A. L., CRUZ I. B. M. Transição epidemiológica, estresse oxidativo e doenças crônicas não transmissíveis sob uma perspectiva evolutiva. Scientia Medica. Porto Alegre, volume 21, número 2, p. 69- 80, 2011.

5. THAPA A. AND CARROLL N. J. Dietary Modulation of Oxidative Stress in Alzheimer's Disease. International Journal of Molecular Sciences, pg. 1-14, 2017.

6. RICHARD F. LOESER, MD. The Role of Aging in The Development Of Osteoarthritis. Transactions of The American Clinical and Climatological Association, vol. 128, pg. 1-11, 2017.

7. SANTOS Z. A. Envelhecimento: a influência do exercício físico nas alterações moleculares associadas ao stress oxidativo em mulheres. Vila real, Universidade De Tras-Os-Montes e Alto Douro, 2014.

8. SKIBSKA B. and GORACA A. The Protective Effect of Lipoic Acid on Selected Cardiovascular Diseases Caused by Age-Related Oxidative Stress. Hindawi Publishing Corporation Oxidative Medicine and Cellular Longevity, Arti- 
cle ID 313021, pg 1-11, 2015.

9. JUNZHEN WE, SHIJIN XIA, BILL KALIONIS, WENBIN WAN, and TAO SUN.; The Role of Oxidative Stress and Inflammation in Cardiovascular Aging. Hindawi Publishing Corporation BioMed Research International, Article ID 615312, pg. 1-13, 2014.

10. MIRELLI PAPALIA DOS SANTOS. O papel das vitaminas antioxidantes na prevenção do envelhecimento cutâneo. UNIJUÍ, 2013.

11. DORIA E., BUONOCORE D., FOCARELLI A., and MARZATICO F. Relationship between Human AgingMuscle and Oxidative System Pathway. Hindawi Publishing Corporation Oxidative Medicine and Cellular Longevity, Article ID 830257, pg. 1-13, 2012.

12. BLASIAK J., PIECHOTA M., PAWLOWSKA E., SZATKOWSKA M., SIKORA E., and KAARNIRANTA K. Cellular Senescence inAge-Related Macul.

13. PASCUAL-AHUIR A., MANZANARES-ESTREDER S., and PROFT M. Pro- and Antioxidant Functions of the Peroxisome-Mitochondria Connection and Its Impact on Aging and Disease. Hindawi Oxidative Medicine and Cellular Longevity, Article ID 9860841, pg. 1-17, 2017.

14. CHANDRASEKARAN A., IDELCHIK M. D. P. S., MELENDEZ A. J. Redox; control of senescence and age-related disease. Redox Biology, vol. 11, pg. 1-12, 2017. 